\title{
Star cluster disruption
}

\author{
Mark Gieles \\ European Southern Observatory, Casilla 19001, Santiago, Chile \\ email: mgieles@eso.org
}

\begin{abstract}
Star clusters are often used as tracers of major star-formation events in external galaxies as they can be studied out to much greater distances than individual stars. It is vital to understand their evolution if they are used to derive, for example, the star-formation history of their host galaxy. More specifically, we want to know how cluster lifetimes depend on their environment and on structural properties such as mass and radius. This review presents a theoretical overview of the early evolution of star clusters and the consequent long-term survival chances. It is suggested that clusters forming with initial densities of $\gtrsim 10^{4} \mathrm{M}_{\odot} \mathrm{pc}^{-3}$ survive the gas expulsion, or 'infant mortality,' phase. At $\sim 10 \mathrm{Myr}$, they are bound and have densities of $\sim 10^{3 \pm 1} \mathrm{M}_{\odot} \mathrm{pc}^{-3}$. After this time, they are stable against expansion through stellar evolution, encounters with giant molecular clouds and will most likely survive for another Hubble time if they are located in a moderate tidal field. Clusters with lower initial densities $\left(\lesssim 100 \mathrm{M}_{\odot} \mathrm{pc}^{-3}\right)$ will disperse into the field within a few 10s of Myrs. Some discussion is given on how extragalactic star cluster populations, and especially their age distributions, can be used to gain insight into disruption.
\end{abstract}

Keywords. galaxies: star clusters, open clusters and associations: general

\section{Introduction}

In the Milky Way, the majority of star formation occurs in embedded clusters (Lada \& Lada 2003). At the same time, only a small fraction of stars in the disc resides in (open) clusters, indicating that most of the clusters/associations are not long-lived and disruption on timescales on the order of the ages of open clusters is important.

Historically, studies of the lifetimes of star clusters focussed on the open clusters in the Milky Way. At the end of the 1950s, the scarcity of old ( $\gtrsim$ Gyr) open clusters was noted nearly simultaneously in three studies: van den Bergh (1957), von Hoerner (1958) and Oort (1958). It was already speculated then by these authors that this was due to their finite lifetimes. The first quantitative explanation for this observation came from Spitzer (1958), who showed that interactions with giant molecular clouds (GMCs) are a plausible cause for the disruption of open clusters. In his model, a cluster with a density of $1 \mathrm{M}_{\odot} \mathrm{pc}^{-3}$ survives the periodic heating due to passing GMCs for about $200 \mathrm{Myr}$. Wielen (1971) constructed an age distribution of open clusters within $1 \mathrm{kpc}$ of the Sun and derived a median lifetime of $200 \mathrm{Myr}$. Because of this remarkable agreement with the theoretical predictions of Spitzer, GMC encounters have since been held as the dominant disruption mechanism for open clusters. Another supporting argument for this idea came from the fact that the old open clusters are strongly concentrated towards the Galactic anticentre, where the density of GMCs is low, and their survival chances high (van den Bergh \& McClure 1980 and also Figure 1).

Later, Elson \& Fall (1985) and Hodge (1987) constructed age distributions for the Large and Small Magellanic Cloud (LMC and SMC) clusters, respectively. They compared both to Wielen's result and showed that the age distributions in the Magellanic Clouds extend 


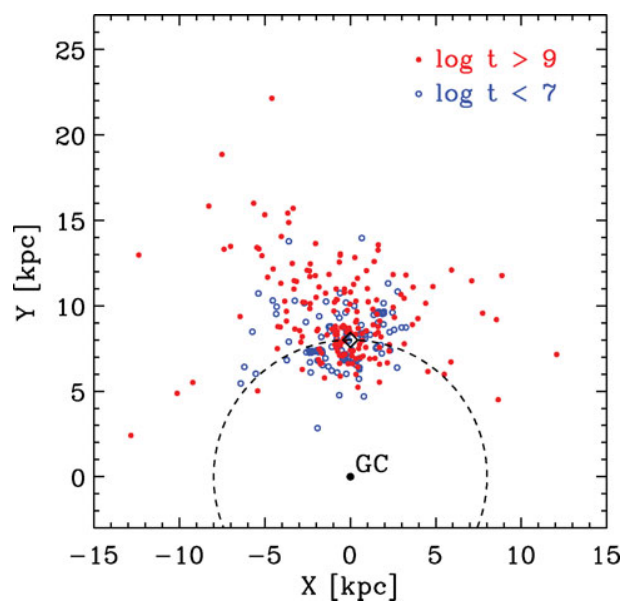

Figure 1. Distribution of young and old open clusters in the Galactic plane, based on the catalogue of Dias et al. (2002). The old open clusters ( $\geqslant 1$ Gyr) are found preferentially towards the Galactic anticentre.

to older ages, and consequently concluded that these clusters survive longer than their Milky Way counterparts.

This review attempts to summarise work done on the disruption of star clusters since the seminal studies mentioned above. Three time spans are considered in which different destruction mechanisms are important:

- First few Myr: expulsion of residual gas from the star-formation process;

- First few 100 Myr: mass loss due to stellar evolution;

- First Gyr: interactions with GMCs and two-body relaxation in a tidal field.

These points will be discussed from a theoretical and observational point of view in Sections 2 and 3, respectively.

\section{Theoretical considerations}

\subsection{The first few Myr: gas expulsion and 'infant mortality' of clusters}

A lot of attention has recently been paid to the disruption of star clusters just after their formation, due to the removal of residual gas from the star-formation process by feedback from hot stars. This was already considered by Tutukov (1978) and Lada \& Lada (2003) argue it could be the explanation for the strong drop at a few Myr in the age distribution of Galactic embedded and open clusters. The arguments as to why this is destructive are as follows: if the embedded stars are in virial equilibrium with their surroundings, then the stellar velocities are too high once the gas is removed. The cluster consequently expands or even completely dissolves. If we define the total mass of the system as $M_{\text {tot }}=M_{\mathrm{g}}+M_{*}$, where $M_{*}$ is the stellar mass and $M_{\mathrm{gas}}$ the gas mass, virial equilibrium before gas expulsion can be written as

$$
v_{\mathrm{rms}}^{2}=\frac{G M_{\mathrm{tot}}}{2 r_{\mathrm{v}}} .
$$

Here, $G$ is the gravitational constant, $v_{\mathrm{rms}}$ the rms velocity of the stars and $r_{\mathrm{v}}$ the virial radius of the (embedded) cluster. The latter relates to the half-mass radius as $r_{\mathrm{v}} \approx 1.25 r_{\mathrm{h}}$, slightly dependent on the density profile. Since $v_{\mathrm{rms}}^{2}$ is three times the one-dimensional velocity dispersion squared, $\sigma_{1 \mathrm{~d}}^{2}$, and $r_{\mathrm{h}}$ is $4 / 3$ times the half-mass radius in projection, 
$r_{\text {eff }}$, Equation (2.1) can also be written in observable quantities as

$$
\sigma_{1 \mathrm{~d}}^{2}=\frac{G M_{\mathrm{tot}}}{\eta r_{\mathrm{eff}}},
$$

with $\eta \approx 10$. In most models it is assumed that the gas-expulsion timescale, $t_{\mathrm{exp}}$, is much shorter than the crossing time of the stars, $t_{\mathrm{cr}}$. The latter is defined as

$$
\begin{aligned}
t_{\mathrm{cr}} & =2 \sqrt{2}\left(\frac{r_{\mathrm{v}}^{3}}{G M}\right)^{1 / 2} \\
& \approx 0.65 \mathrm{Myr}\left(\frac{\rho_{\mathrm{h}}}{10^{3} \mathrm{M}_{\odot} \mathrm{pc}^{-3}}\right)^{-1 / 2} .
\end{aligned}
$$

For $t_{\mathrm{cr}} \gg t_{\exp }$ the positions of the stars are static during the change of the potential and the cluster's response can be calculated using the impulsive approximation.

The velocities of the stars right after gas removal are still defined by the total mass of the embedded cluster (Equation 2.1) and hence the cluster will expand to find a new equilibrium. Let's take $M_{*}=\epsilon M_{\text {tot }}$, such that $\epsilon$ is the star-formation efficiency (SFE). The final radius can then be expressed in terms of $\epsilon$ and the initial radius, $r_{\mathrm{v}}(0)$, as (e.g., Hills 1980)

$$
\frac{r_{\mathrm{v}}}{r_{\mathrm{v}}(0)}=\frac{\epsilon}{2 \epsilon-1}
$$

From this, it can be seen that for $\epsilon \leqslant 0.5$ the cluster dissolves. Since this value is high for a high SFE, gas expulsion is a plausible cause for the disruption of a large fraction of embedded clusters. A value of $\epsilon=0.5$, however, is (for several reasons) an upper limit for complete destruction of the cluster:

- Escape of unbound stars: For $\epsilon \leqslant 0.5$, the system is globally unbound since the total energy (kinetic plus potential) is positive. However, most of the positive energy is carried away by escaping stars, which have velocities much higher than $v_{\text {rms }}$. Almost all dynamical models find that for $\epsilon \gtrsim 0.33$ a fraction of the stars will remain bound (see Figure 2).

- Subvirial initial conditions: This was first discussed by Lada et al. (1984) who considered a collapsing cloud in which the stars are dynamically 'cold' while the gas is removed (see also Proszkow et al. 2009). If the stellar velocities are only a fraction, $f$, of the virial velocities then Equation $(2.5)$ becomes $r_{\mathrm{v}} / r_{\mathrm{v}}(0)=\epsilon /\left(2 \epsilon-f^{2}\right)$, i.e., the condition for complete disruption becomes $\epsilon=f^{2} / 2$, which for $f=0.5$ is $\epsilon=0.125$ (Goodwin 2008), much lower than the analytical estimate of $\epsilon=0.5$ for initially virialised, embedded clusters. The combination of $\epsilon$ and the initial virial state, or the effective SFE (Goodwin \& Bastian 2008), thus determines the survival chance.

- Clumpy initial conditions: Fellhauer et al. (2009) showed that if stars form in a clumpy fashion, and if these clumps merge quickly, then the survival chance of the merger remnant is enhanced.

- Slow gas removal: If the gas-expulsion timescale is longer than $t_{\mathrm{cr}}$, the cluster will expand adiabatically, which can result in a bound cluster for $\epsilon$ as low as 0.1 (e.g., Geyer \& Burkert 2001; Baumgardt \& Kroupa 2007 and Figure 2).

Therefore, the importance of the gas-removal phase in disrupting star clusters depends on many parameters: the initial density of the embedded cluster, the gas-removal timescale, the initial virial state of the embedded cluster, the star-formation efficiency and the initial configuration of the stars (clumpy or smooth density profile). This has led to several different predictions. For example, most models assume that gas removal is instantaneous. If $\epsilon$ is the same for all clusters then the disruption rate is independent 


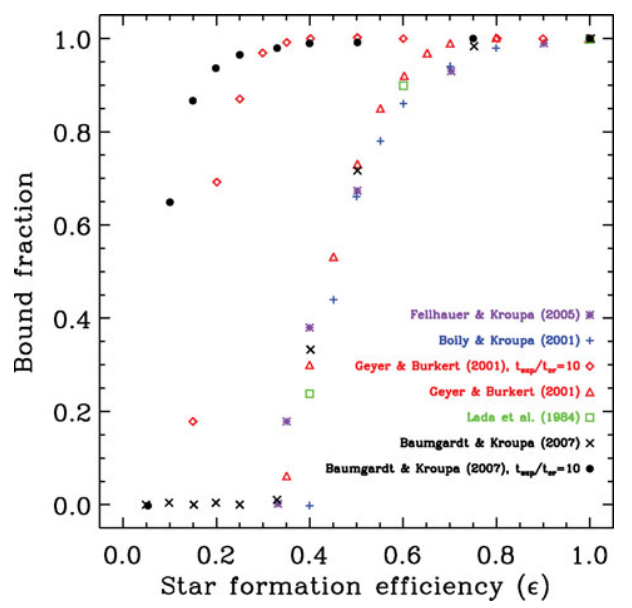

Figure 2. Fraction of stellar mass that remains bound after gas expulsion for different SFEs, found by several authors using $N$-body simulations. (Data taken from Baumgardt \& Kroupa 2007.)

of cluster mass. Baumgardt et al. (2008) advocate, however, that gas expulsion is less efficient in disrupting massive clusters because of their higher binding energy per unit mass. This makes the general prediction that the exposed cluster mass function should be shallower than the embedded cluster mass function (see Parmentier et al. 2008).

Since the embedded phase is brief $(\sim 1 \mathrm{Myr})$, most of these parameters are poorly constrained from observations and the interpretation of the models is therefore sensitive to the assumptions that are made with regard to the initial conditions. In Section 3 possible observational signatures of this early disruption will be discussed.

\subsection{The first few 100 Myr: mass loss due to stellar evolution}

Clusters that survive the gas-expulsion phase continue to lose mass through the evolution of the member stars. For a Kroupa-type initial mass function (IMF) between 0.1 and $100 \mathrm{M}_{\odot}$, the cluster mass reduces roughly by $10 / 20 / 30 \%$ in the first $10 / 100 / 500 \mathrm{Myr}$. Since these stellar evolution timescales are much longer than typical values of $t_{\mathrm{cr}}$, the cluster will, in most cases, react by adiabatic expansion. This expansion is analogous to the increase of the orbital separation of a binary when one of the members loses mass. It is far less sensitive to the fractional mass loss than in the impulsive approximation, since

$$
\begin{aligned}
\frac{r_{\mathrm{v}}(t)}{r_{\mathrm{v}}(0)} & =\frac{M(0)}{M(t)} \\
& =\frac{1}{\mu} .
\end{aligned}
$$

In the last step, $\mu$ is used for the fraction of mass that remains and this can be compared to $\epsilon$ in the instantaneous-mass-loss case (Equation 2.5). For $\mu=0.5$, the cluster will expand by a factor of two and not lose mass, whereas for impulsive mass loss the cluster nearly dissolves, as we saw in Section 2.1.

The situation is in reality somewhat more complicated than this simple prediction. The expansion is more severe and can even result in complete disruption if the cluster is mass segregated before the bulk of the stellar evolution takes place (Vesperini et al. 2009). If the cluster is not primordially mass segregated, it will still segregate while the stars evolve (Applegate 1986) and this can eventually lead to enhanced expansion at later times. Finally, the fate of the cluster also depends on the Jacobi (or tidal) radius $\left(r_{\mathrm{J}}\right)$. 
The combined effect of mass loss through stellar evolution and dynamical evolution in a tidal field was considered by Fukushige \& Heggie (1995). They show that when clusters expand to a radius of $\sim 0.5 r_{\mathrm{J}}$, they lose equilibrium and most of their stars overflow $r_{\mathrm{J}}$ in a few crossing times. As was the case with gas expulsion, a lot more observational constraints on the initial conditions of star clusters, such as the degree of primordial mass segregation and the initial radius with respect to the tidal radius, are needed before we can make general statements about the effect of stellar evolution on the evolution of star clusters.

\subsection{Interaction with giant molecular clouds}

An external disruptive factor, already considered by Spitzer (1958), is represented by cluster encounters with GMCs. Since GMCs are typically more massive than clusters, the cluster is more affected by an encounter than the cloud (Theuns 1991). The cluster lifetime due to periodic heating of passing clouds is inversely proportional to the volume density of molecular gas, $\rho_{\text {gas }}$, and proportional to the density of the cluster, a typical result for disruption by external tidal 'shocks' (e.g., Ostriker et al. 1972)

$$
t_{\mathrm{dis}}^{\mathrm{GMC}} \approx 1 \mathrm{Gyr}\left(\frac{\rho_{\mathrm{gas}}^{\odot}}{\rho_{\mathrm{gas}}}\right)\left(\frac{\rho_{\mathrm{h}}}{10 \mathrm{M}_{\odot} \mathrm{pc}^{-3}}\right) .
$$

Here, $\rho_{\text {gas }}^{\odot} \approx 0.03 \mathrm{M}_{\odot} \mathrm{pc}^{-3}$ is the molecular-gas density in the solar neighbourhood and the constant is taken from Gieles et al. (2006) and is equal, within a factor of two, to the original result of Spitzer (1958). The dependence of $t_{\mathrm{dis}}$ on $\rho_{\mathrm{gas}}$ means that lifetimes of star clusters also scale roughly inversely with the observable surface density of molecular gas, $\Sigma_{\text {gas }}$, enabling us to make order-of-magnitude predictions for the lifetimes of clusters in other galaxies. In spiral galaxies, disruption by GMC encounters is especially important in the early stages of evolution, since clusters form in the thin gaseous disc where $\rho_{\text {gas }}$ is high. Older clusters are typically more associated with the thick disc, where $\rho_{\text {gas }}$ is low and GMC encounters are less frequent. Since young $(\lesssim 1 \mathrm{Gyr})$ clusters in spiral galaxies have a near-constant radius (e.g., Larsen 2004), Gieles et al. (2006) argue that the dissolution time due to GMC encounters is longer for more massive clusters, due to their higher density. It is not clear whether this lack of a mass-radius relation is a universal property imprinted by formation, or the result of an evolutionary effect. This will be addressed in Section 3 .

\subsection{Two-body relaxation in the tidal field of the host galaxy}

On somewhat longer timescales, stars are lost from clusters through two-body relaxation in which stars get accelerated to the escape velocity, $v_{\text {esc }}$ (e.g., Ambartsumian 1938; Spitzer 1940). The relevant timescale of escape is, to first order, the half-mass relaxation time (Spitzer 1987),

$$
t_{\mathrm{rh}}=0.138 \frac{N^{1 / 2} r_{\mathrm{h}}^{3 / 2}}{\sqrt{\bar{m} G} \ln \Lambda},
$$

where $N$ is the number of stars, $\bar{m}$ the mean stellar mass and $\ln \Lambda$ the Coulomb logarithm: $\ln \Lambda \approx \ln 0.11 N$ (Giersz \& Heggie 1994). The relaxation time is approximately the time stars need to establish a Maxwellian velocity distribution. A fraction $\xi_{\mathrm{e}}$ of the stars in the tail of the distribution have velocities larger than $v_{\text {esc }}$ and consequently escape. Assuming that this high-velocity tail is refilled every $t_{\mathrm{rh}}$, the dissolution timescale is $t_{\mathrm{dis}}=t_{\mathrm{rh}} / \xi_{\mathrm{e}}$. For isolated clusters, $v_{\mathrm{esc}}=2 v_{\mathrm{rms}}$. For a Maxwellian velocity distribution a fraction $\xi_{\mathrm{e}}=0.0074$ has $v>2 v_{\mathrm{rms}}$ and then $t_{\mathrm{dis}}=137 t_{\mathrm{rh}}$. For tidally limited clusters $\xi_{\mathrm{e}}$ is higher since $v_{\mathrm{esc}}$ is lower. For a typical cluster density profile $\xi_{\mathrm{e}} \approx 0.033$, implying 
$t_{\mathrm{dis}} \approx 30 t_{\mathrm{rh}}$ (Spitzer 1987). The escape fraction $\xi_{\mathrm{e}}$ is often taken as constant (e.g., Gnedin \& Ostriker 1997). However, $\xi_{\mathrm{e}}$ depends on $r_{\mathrm{h}}$ (through $v_{\mathrm{rms}}$ ) and on the strength of the tidal field, or $r_{\mathrm{J}}$ (through $v_{\mathrm{esc}}$ ). Effectively, $\xi_{\mathrm{e}}$ depends on the ratio $r_{\mathrm{h}} / r_{\mathrm{J}}$ (e.g., Spitzer \& Chevalier 1973; Wielen 1988). Gieles \& Baumgardt (2008) show that $\xi_{\mathrm{e}} \propto\left(r_{\mathrm{h}} / r_{\mathrm{J}}\right)^{3 / 2}$ for $r_{\mathrm{h}} / r_{\mathrm{J}} \gtrsim 0.05$ (the tidal regime). Combined with Equation (2.9), we then find for clusters on circular orbits in the tidal regime that $t_{\mathrm{dis}} \propto N / \omega$, apart from the slowly varying Coulomb logarithm. Here, $\omega \equiv V_{\mathrm{G}} / R_{\mathrm{G}}$ is the angular frequency in the galaxy and $R_{\mathrm{G}}$ and $V_{\mathrm{G}}$ are the galactocentric distance and the velocity around the galaxy centre, respectively. So, for a flat rotation curve, we find $t_{\mathrm{dis}} \propto R_{\mathrm{G}}$ for a cluster of a given mass (e.g., Chernoff \& Weinberg 1990; Vesperini \& Heggie 1997). This linear dependence of $t_{\text {dis }}$ on $R_{\mathrm{G}}$ makes it difficult to explain the universality of the globular cluster mass function from dynamical evolution of a power-law initial cluster mass function (e.g., Vesperini et al. 2003), but this will not be discussed here. Note that $\omega$ can also be written as $\omega \propto \rho_{\mathrm{J}}^{1 / 2}$, where $\rho_{\mathrm{J}}$ is the density within $r_{\mathrm{J}}$, which is how Lee \& Ostriker (1987) present it.

Fukushige \& Heggie (2000) show that the linear scaling of $t_{\text {dis }}$ with $N$ is slightly affected by the finite time it takes stars to find the Lagrangian 'exit' points. Baumgardt (2001) showed that $t_{\mathrm{cr}}$ is of importance and finds, for equal-mass clusters, that $t_{\mathrm{dis}} \propto t_{\mathrm{rh}}^{3 / 4} t_{\mathrm{cr}}^{1 / 4}$. Baumgardt \& Makino (2003) find that this scaling also holds for models of clusters with a mass spectrum, stellar evolution and for different types of orbits in a logarithmic potential. Their result for $t_{\text {dis }}$ can be summarised as

$$
t_{\mathrm{dis}} \approx 2 \operatorname{Myr}\left(\frac{N}{\ln \Lambda}\right)^{3 / 4} \frac{R_{\mathrm{G}}}{\mathrm{kpc}}\left(\frac{V_{\mathrm{G}}}{220 \mathrm{~km} \mathrm{~s}^{-1}}\right)^{-1}(1-\varepsilon),
$$

where $\varepsilon$ is the eccentricity of the orbit. If the Coulomb logarithm is taken into account, the scaling is something like $t_{\mathrm{dis}} \propto N^{0.65}$ (Lamers et al. 2005).

\section{Observational signatures of cluster disruption}

\subsection{The velocity dispersion of young clusters}

Ho \& Filippenko (1996) were the first to demonstrate that one can empirically determine the dynamical mass, $M_{\mathrm{dyn}}$, of star clusters beyond the Local Group using their integrated light. They used high-resolution spectroscopy to measure $\sigma_{1 \mathrm{~d}}$ from the width of the spectral lines and high-angular-resolution (HST) imaging to measure $r_{\text {eff }}$. The dynamical mass then follows from the virial relation (Equation 2.2). They did this for the $\sim 10 \mathrm{Myr}-$ old super star cluster 'A' in the dwarf starburst galaxy NGC 1569 and found that its $M_{\text {dyn }}$ is comparable to the masses of Milky Way globular clusters, concluding that cluster 'A' is a young globular cluster. Since then, this analysis has been applied to several dozen more clusters with different ages and masses and in different galaxies (e.g., Larsen et al. 2004; Bastian et al. 2006; McCrady \& Graham 2007; Mengel et al. 2008).

\subsection{Supervirial and dispersing?}

The empirically derived $M_{\text {dyn }}$ relies on the assumption that the cluster is in virial equilibrium. One way of verifying the validity of this assumption is by comparing $M_{\mathrm{dyn}}$ with the photometric mass, $M_{\text {phot }}$. The latter can be derived from the cluster's total luminosity and age and comparison to an SSP model. Bastian et al. (2006) provide a compilation of $M_{\mathrm{phot}}$ and $M_{\mathrm{dyn}}$ values of 19 clusters. They find that for the somewhat older clusters $(\gtrsim 50-100 \mathrm{Myr})$, there is good agreement between $M_{\text {phot }}$ and $M_{\mathrm{dyn}}$. For many of the young $(\sim 10 \mathrm{Myr})$ star clusters $M_{\mathrm{dyn}} \gg M_{\mathrm{phot}}$. Goodwin \& Bastian (2006) suggest 

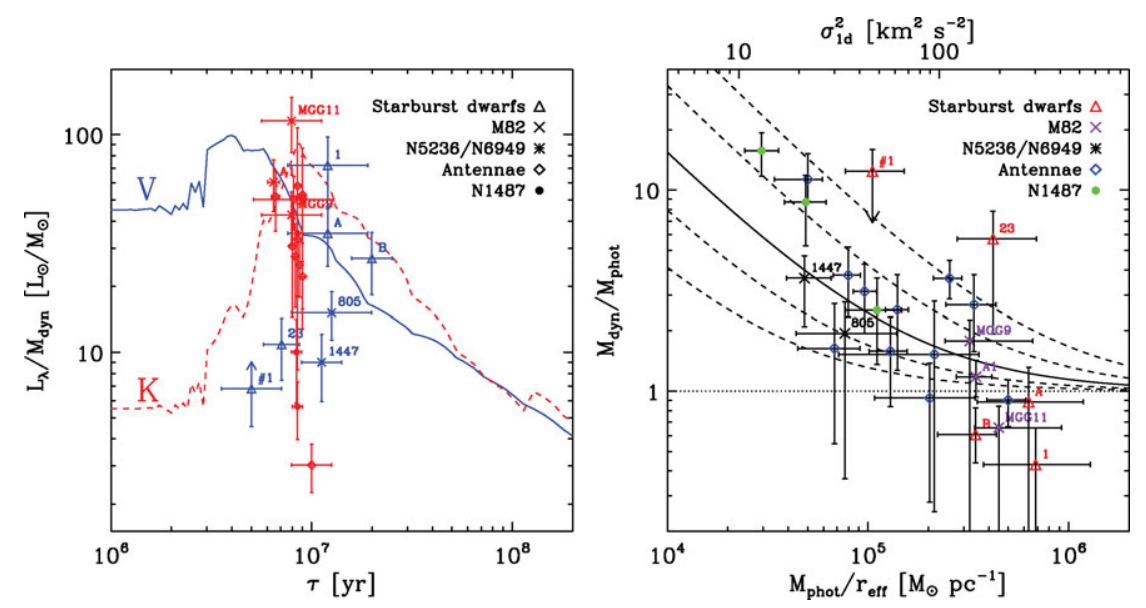

Figure 3. (left) Light-to-dynamical-mass ratio for 24 clusters from the literature. The red and blue points refer to studies done in the optical and near-infrared, respectively. Different symbols correspond to different galaxy types. The lines show the photometric evolution of an SSP model in two filters. (right) Dynamical mass over photometric mass for the same clusters, shown as a function of $M_{\text {phot }} / r_{\text {eff }}$, which is a proxy of $\sigma_{1 \mathrm{~d}}$ (Equation 2.2). The full line is a prediction of the effect of binaries on $M_{\mathrm{dyn}}$ (Equation 3.1), with $1 \sigma$ and $2 \sigma$ variations due to stochastic fluctuations shown as dashed lines. (Figure from Gieles et al. 2009.)

that this is a signature of gas expulsion and the 'infant mortality' scenario discussed in Section 2.1, i.e., they conclude that these clusters are supervirial.

In the left panel of Figure 3, an updated version of figure 5 in Bastian et al. (2006) is given. It shows the light-to-dynamical-mass ratio of 24 young star clusters in different galaxies. The data are compiled from the literature (McCrady et al. 2003 ; Bastian et al. 2006; Smith et al. 2006; McCrady \& Graham 2007; Moll et al. 2007; Östlin et al. 2007; Larsen et al. 2008; Mengel et al. 2008) and will be discussed in more detail in Gieles et al. (2009) and Portegies Zwart et al. (2010). The observations taken in the visible are shown in blue and those taken in the near-infrared are shown in red. The photometric evolution in the $V$ and $K$ bands from the Bruzual \& Charlot (2003) SSP models using a Chabrier IMF is shown as a full (blue) line and a dashed (red) line, respectively. All clusters have an age close to $10 \mathrm{Myr}$. This is because of observational constraints: younger clusters are more obscured and at $10 \mathrm{Myr}$ the red-supergiant phase sets in, making the clusters brighter (especially in the near-infrared; see Figure 3) and therefore easier to study.

It is important to realise what initial conditions are required for the gas-expulsion scenario to work. The models discussed in Section 2.1 show that after instantaneous gas removal it takes roughly 10 initial crossing times to either completely disrupt or expand to a new virial equilibrium. In the models of Goodwin \& Bastian (2006) this corresponds to $\sim 25 \mathrm{Myr}$ since their clusters have $t_{\mathrm{cr}}=2.5 \mathrm{Myr}$ in the embedded phase. Only with an initial $t_{\mathrm{cr}}$ of a few Myr is it possible to 'catch' an unbound, expanding cluster at $10 \mathrm{Myr}$. This corresponds to an initial density (stars and gas) of $\sim 100 \mathrm{M}_{\odot} \mathrm{pc}^{-3}$ (Equation 2.4). Since the clusters considered here have densities of $\sim 10^{3} \mathrm{M}_{\odot} \mathrm{pc}^{-3}$ (see Figure 4), gas expulsion cannot be responsible for the high velocity dispersions. The densities in the embedded phase were at least a factor $1 / \epsilon$ higher because of the presence of gas, but probably even more since they have also expanded since then. A similar conclusion can be drawn from the measured velocities, which are on the order of $20 \mathrm{~km} \mathrm{~s}^{-1}$. With such velocities, an unbound cluster dissolves into the field in a few Myr, incompatible with their radii being only a few pc. We conclude that the 'infant mortality' phase of dense clusters 
only lasts a few Myr. The clusters considered here have evolved for at least $10-100 t_{\mathrm{cr}}$ and are therefore probably bound objects (an argument also made by McCrady and collaborators).

\subsection{Binaries}

The point remains that the measured velocity dispersions are too high for some of the clusters. It could be that the IMF assumption is invalid. The virial velocities are higher when the IMF is more bottom-heavy, but the differences between the measured and the virial velocities are probably too large to be explained by IMF variations. An alternative is that the velocities are dominated by orbital motions of binaries in the cluster, not taken into account in the virial relation (Equation 2.2). Kouwenhoven \& de Grijs (2008) considered this and they show that the $M_{\mathrm{dyn}} / M_{\text {phot }}$ ratio can be elevated by several factors (see also de Grijs, these proceedings). But they concluded that the contribution of binaries to the velocity dispersion is less than $5 \%$ for clusters with $\sigma_{1 \mathrm{~d}} \gtrsim 10 \mathrm{~km} \mathrm{~s}^{-1}$. So binaries cannot be responsible for the observed velocities according to their model. But Kouwenhoven \& de Grijs (2008) did not include the mass-dependent stellar mass-to-light ratio in their models and also did not take into account stars more massive than $20 \mathrm{M}_{\odot}$. Therefore, the effect will be stronger for the clusters considered in Figure 3, since the light is dominated by massive stars $\left(\sim 15 \mathrm{M}_{\odot}\right)$ for which (primordial) multiplicity is high and short periods and mass ratios close to unity are common.

Gieles et al. (2009) derive a simple analytic expression for the additional velocity dispersion due to orbital motions of stars in binaries, $\sigma_{\text {bin }}$, relative to the dynamical dispersion of a virialised cluster, $\sigma_{1 \mathrm{~d}}$,

$$
\frac{\sigma_{\text {bin }}^{2}}{\sigma_{1 \mathrm{~d}}^{2}} \approx\left(\frac{f}{0.25}\right)\left(\frac{q}{0.6}\right)^{\frac{3}{2}}\left(\frac{m_{1}}{15 \mathrm{M}_{\odot}}\right)^{\frac{2}{3}}\left(\frac{10^{3} \text { days }}{P}\right)^{\frac{2}{3}}\left(\frac{M / r_{\mathrm{eff}}}{10^{5} \mathrm{M}_{\odot} \mathrm{pc}^{-1}}\right)^{-1} .
$$

Here, $f$ is the binary fraction, $q$ the mass ratio, $m_{1}$ the mass of the primary star (the contribution of the secondary star is ignored) and $P$ the orbital period. The normalisation constants are based on properties of massive binaries in the Milky Way and appropriate for an age of $10 \mathrm{Myr}$. Since the cluster light is dominated by the most massive stars, the measured velocity dispersion is affected by binaries according to this relation. The prediction for $M_{\mathrm{dyn}}$ including the velocities of binaries $\left(M_{\mathrm{dyn}} \propto \sigma_{1 \mathrm{~d}}^{2}+\sigma_{\text {bin }}^{2}\right)$ is shown in the right panel of Figure 3 (full line). The dashed lines show the $1 \sigma$ and $2 \sigma$ spread in the model due to stochastics. The discrepancy between $M_{\text {dyn }}$ and $M_{\text {phot }}$ can largely be explained by virialised clusters with a modest binary population. Assuming that all clusters host a similar binary population, the clusters with a low dynamical dispersion, for which we use $M_{\text {phot }} / r_{\text {eff }}$ as a proxy (Equation 2.2), are affected most. This could be the reason why the data show a decreasing $M_{\mathrm{dyn}} / M_{\text {phot }}$ ratio for an increasing $M_{\mathrm{phot}} / r_{\mathrm{eff}}$ ratio.

This suggests that these clusters are survivors of the gas-expulsion phase and are at the beginning of the stellar evolution phase. In the next Hubble time, these clusters will adiabatically expand by a factor of $\sim 2$ due to mass loss through stellar evolution if they are not mass segregated (Section 2.2) and it remains to conclude that these clusters are good young globular cluster candidates if the tidal field they are in is not too strong.

\subsection{The mass-radius relation of star clusters}

Several studies have pointed out that there is a very weak dependence of cluster radius on mass/luminosity (e.g., Zepf et al. 1999; Larsen 2004; Bastian et al. 2005). For the clusters in Figure 4, this seems not to be the case. Although these are only 24 clusters, and it is far from a tight relation, these objects seem more consistent with a constant 




Figure 4. Mass-radius relation for the same 24 clusters shown in Figure 3, using the same colour and symbol coding and $r_{\mathrm{h}}=(4 / 3) r_{\mathrm{eff}}$. Lines of constant half-mass density, $\rho_{\mathrm{h}} \equiv 3 M /\left(8 \pi r_{\mathrm{h}}^{3}\right)$, are overplotted. The cluster densities imply $0.2 \lesssim t_{\mathrm{cr}} / \mathrm{Myr} \lesssim 2$ (Equation 2.4 ).

density of $\rho_{\mathrm{h}} \approx 10^{3 \pm 1} \mathrm{M}_{\odot}$, or $r_{\mathrm{h}} \approx\left(M / 10^{4} \mathrm{M}_{\odot}\right)^{1 / 3}$, within a factor of two. The difference between this figure and the studies mentioned above is that the clusters considered here are confined to a narrow (young) age range. The mass-radius relation is of importance for disruption since $t_{\mathrm{dis}}^{\mathrm{GMC}} \propto \rho_{\mathrm{h}}$ (Section 2.3). So, for a constant cluster density, GMCs will destroy all clusters equally fast. If the mass-radius relation evolves with time, for example to a near-constant radius as found by several authors, then $t_{\mathrm{dis}}^{\mathrm{GMC}}$ will become mass dependent. In any case, the densities of these clusters imply that $t_{\text {dis }}^{\text {GMC }}$ is much longer than a Hubble time, even when $\rho_{\text {gas }}$ is 10 times higher than in the solar neighbourhood (Equation 2.8), so GMC encounters will not play an important role in the disruption of these clusters at this stage.

In a solar-neighbourhood-type tidal field all clusters in Figure 4 have $r_{\mathrm{h}} / r_{\mathrm{J}} \approx 0.03$ within a factor of two. This implies that these clusters are probably also very stable against expansion due to mass loss through stellar evolution, since in Section 2.2 it was argued that rapid dissolution due to mass loss through stellar evolution only occurs when $r_{\mathrm{h}} / r_{\mathrm{J}}$ increases to $\sim 0.5$. A similar idea was recently put forward by Pfalzner (2009), who discovered two evolutionary sequences in young Galactic star clusters. A dense group that starts with a density of $\sim 10^{5} \mathrm{M}_{\odot} \mathrm{pc}^{-3}$ at a few Myr containing the Arches cluster, NGC 3603 and Trumpler 14, to which they refer as 'starburst clusters.' They seem to expand along a sequence of constant mass, where at $10-20 \mathrm{Myr}$ there are the redsupergiant clusters (RSGC01 and RSGC02) with a density of $\sim 10^{3} \mathrm{M}_{\odot} \mathrm{pc}^{-3}$. The second sequence also starts at a few Myr, but at much lower densities $\left(\sim 10 \mathrm{M}_{\odot} \mathrm{pc}^{-3}\right)$ and expands along a $M \propto 1 / r_{\mathrm{h}}$ sequence to densities comparable to the field-star density. These are referred to as 'leaky clusters.' The clusters in Figure 4 could be compared to the red-supergiant clusters in the Milky Way, i.e., the end stage of the starburst sequence.

\section{Discussion}

Until the 1990s, the open clusters in the Milky Way and the populous clusters in the Magellanic Clouds were the targets of studies of disruption. HST has enabled us to establish the properties of larger populations of clusters containing more massive clusters in quiescent spiral galaxies (e.g., Larsen \& Richtler 2000), interacting galaxies 


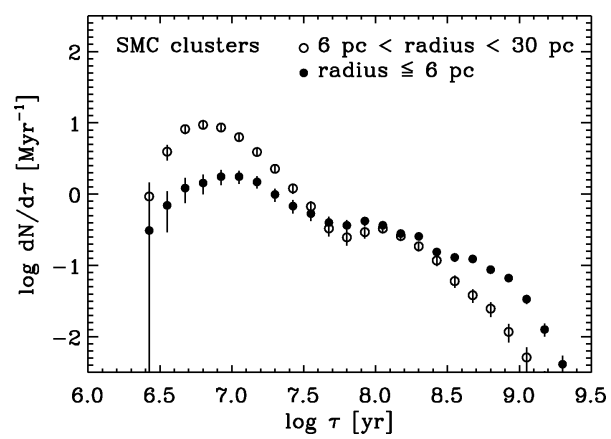

Figure 5. Age distribution of SMC clusters based on the catalogue of Chiosi et al. (2006). The sample is split into small and large clusters/associations, with the boundary at $6 \mathrm{pc}$. The histograms are made using a 0.5 dex bin width with different starting values (boxcar averaging).

(e.g., Bastian et al. 2005; Whitmore et al. 1999) and merger remnants (e.g., Miller et al. 1997).

The primary tool used for studies of cluster disruption is the age distribution. Different groups give varying weights to the various aspects described in Section 2 in the interpretation of the results. There are two flavours of empirically based cluster disruption models: one is based on externally driven cluster disruption. In this model, the dissolution time depends on the mass of the cluster and its environment. It was based on the age and mass distributions of luminosity-limited cluster samples in different galaxies (Boutloukos \& Lamers 2003). The variations in dissolution timescales are explained by differences in tidal-field strength (Lamers et al. 2005) and GMC density (Gieles et al. 2006). The second model assumes that internally driven cluster disruption is most important. In this model, roughly $80-90 \%$ of the number of clusters is destroyed each age dex resulting in a (mass-limited) age distribution that declines roughly as $\tau^{-1}$. In this model, it is assumed that the infant mortality of clusters proceeds for a few $100 \mathrm{Myr}$ and that the disruption rate is equally fast for all masses. This model is based on the cluster population of the Antennae galaxies (Fall et al. 2005; Whitmore et al. 2007) and it was argued by these authors that their model is universal and should be able to describe the age distributions of cluster populations in other galaxies as well.

The universal cluster dissolution scenario has led to some controversy. Chandar et al. (2006) show that the age distribution of SMC clusters declines as $\tau^{-0.85}$, almost the same as in the Antennae. Gieles et al. (2007) used the same data set and show that the age distribution of massive clusters $\left(\gtrsim 10^{3.5} \mathrm{M}_{\odot}\right)$ is flat in the first few $100 \mathrm{Myr}$. They reproduce the $\tau^{-0.85}$ result for the full sample and conclude that the decline is caused by detection incompleteness, confirmed by de Grijs \& Goodwin (2008) using similar arguments but an independent data set. Boutloukos \& Lamers (2003) show that for a constant formation history and no disruption, the age distribution of a luminosity-limited sample declines as $\tau^{-\zeta(\alpha-1)}$, where $-\alpha$ is the index of the cluster initial mass function. The index $\zeta$ describes the cluster fading with age: $F_{\lambda}(\tau) \propto \tau^{-\zeta}$, with $F_{\lambda}$ being the flux at wavelength $\lambda$ of a cluster with constant mass. For the $U(V)$ band, $\zeta \approx 1.0(0.7)$, resulting in $\tau^{-\eta}$, with $0.7 \lesssim \eta \lesssim 1.0$ due to fading alone when $\alpha=2$. If the cluster mass function is a Schechter function (Gieles 2009; Larsen 2009), the age distribution of a luminosity-limited sample not affected by disruption is as steep as $0.9 \lesssim \eta \lesssim 1.4$ (see also Konstantopoulos, these proceedings). Both models rely on the assumption that the cluster-formation history has been constant in the age range considered. Bastian et al. 
(2009) recently pointed out that this is invalid for interacting galaxies like the Antennae galaxies and that a recent burst can be confused by mass-independent cluster disruption.

Another issue, especially important for distant cluster populations, is what we actually define as a star cluster. Figure 5 shows the age distribution of clusters and associations in the central region of the SMC (Chiosi et al. 2006). The sample is split in two subsamples of $\sim 200$ clusters, based on their size. The age distribution of large clusters falls off much more quickly than that of the compact group. The median radii of both samples are $4.5 \mathrm{pc}$ and $9 \mathrm{pc}$, respectively. At a distance of $20 \mathrm{Mpc}$, it will be very hard to distinguish between these two groups. A speculative suggestion is that more of the short-lived 'associations' or 'leaky clusters' are included in cluster samples in distant galaxies, which would not be considered genuine star clusters if they could be resolved.

\section{Acknowledgement}

I thank Brad Whitmore and Iraklis Konstantopoulos for an interesting discussion over lunch at Copacabana beach during this conference and the organisers of IAUS 266 for an interesting meeting!

\section{References}

Applegate, J. H. 1986, ApJ, 301, 132

Ambartsumian, V. A. 1938, Sci. Mem. Leningrade State Univ. \#22, Ser. Math. Sci., 4, 19

Bastian, N., Gieles, M., Lamers, H. J. G. L. M., Scheepmaker, R. A., \& de Grijs, R. 2005 A\&A, 431, 905

Bastian, N., Saglia, R. P., Goudfrooij, P., Kissler-Patig, M., Maraston, C., Schweizer, F., \& Zoccali, M. 2006, A\&广A, 448, 881

Bastian, N., Trancho, G., Konstantopoulos, I. S., \& Miller, B. W. 2009, ApJ, 701, 607

Baumgardt, H. 2001, MNRAS, 325, 1323

Baumgardt, H. \& Kroupa, P. 2007, MNRAS, 380, 1589

Baumgardt, H., Kroupa, P., \& Parmentier, G. 2008, MNRAS, 384, 1231

Baumgardt, H. \& Makino, J. 2003, MNRAS, 340, 227

Boutloukos, S. G. \& Lamers, H. J. G. L. M. 2003, $M N R A S, 338,717$

Bruzual, G. \& Charlot, S. 2003, MNRAS, 344, 1000

Chandar, R., Fall, S. M., \& Whitmore, B. C. 2006, ApJ (Letters), 650, L111

Chernoff, D. F. \& Weinberg, M. D. 1990, ApJ, 351, 121

Chiosi, E., Vallenari, A., Held, E. V., Rizzi, L., \& Moretti, A. 2006, A\&A, 452, 179

de Grijs, R. \& Goodwin, S. P. 2008, MNRAS, 383, 1000

Dias, W. S., Alessi, B. S., Moitinho, A., \& Lépine, J. R. D. 2002, A $\& A, 389,871$

Elson, R. A. W. \& Fall, S. M. 1985, ApJ, 299, 211

Fall, S. M., Chandar, R., \& Whitmore, B. C. 2005, ApJ (Letters), 631, L133

Fellhauer, M., Wilkinson, M. I., \& Kroupa, P. 2009, MNRAS, 397, 954

Fukushige, T. \& Heggie, D. C. 1995, MNRAS, 276, 206

Fukushige, T. \& Heggie, D. C. 2000, MNRAS, 318, 753

Geyer, M. P. \& Burkert, A. 2001, MNRAS, 323, 988

Gieles, M. \& Baumgardt, H. 2008, MNRAS, 389, L28

Gieles, M., Lamers, H. J. G. L. M., \& Portegies Zwart, S.F. 2007, ApJ, 668, 268

Gieles, M., Portegies Zwart, S. F., Baumgardt, H., Athanassoula, E., Lamers, H. J. G. L. M., Sipior, M., \& Leenaarts, J. 2006, MNRAS, 371, 793

Gieles, M. 2009, MNRAS, 394, 2113

Gieles, M., Sana, H., \& Portegies Zwart, S. F. 2009, MNRAS, in press (arXiv:0911.1557)

Giersz, M. \& Heggie, D. C. 1994, MNRAS, 268, 257

Gnedin, O. Y. \& Ostriker, J. P. 1997, ApJ, 474, 223

Goodwin, S. P 2008, in: E. Pérez, R. de Grijs, \& R. M. González Delgado (eds.), Young massive star clusters - Initial conditions and environments, ApSS, 324, 259 
Goodwin, S. P. \& Bastian, N. 2006, MNRAS, 373, 752

Hills, J. G. 1980, ApJ, 235, 986

Ho, L. C. \& Filippenko, A. V. 1996, ApJ (Letters), 466, L83

Hodge, P. 1987, PASP, 99, 724

Kouwenhoven, M. B. N., \& de Grijs, R. 2008, A\& $\&$, 480, 103

Lada, C. J. \& Lada, E. A. 2003, ARA\&A, 41, 57

Lada, C. J., Margulis, M., \& Dearborn, D. 1984, ApJ, 285, 141

Lamers, H. J. G. L. M., Gieles, M., \& Portegies Zwart, S. F. 2005, A\&\&A, 429, 173

Larsen, S. S. 2004, $A \& A, 416,537$

Larsen, S. S. 2009, $A \& A$, 494, 539

Larsen, S. S., Origlia, L., Brodie, J., \& Gallagher III, J. S. 2008, MNRAS, 383, 263

Larsen, S. S., Brodie, J. P., \& Hunter, D. A. 2004, AJ, 128, 2295

Larsen, S. S., \& Richtler, T. $2000 A \mathscr{S} A, 354,836$

Lee, H. M., \& Ostriker, J. P. 1987, ApJ, 322, 123

McCrady, N., Gilbert, A. M., \& Graham, J. R. 2003, ApJ, 596, 240

McCrady, N. \& Graham, J. R. 2007, ApJ, 663, 844

Mengel, S., Lehnert, M. D., Thatte, N. A., Vacca, W. D., Whitmore, B., \& Chandar, R. 2008, $A \mathscr{E} A, 489,1091$

Miller, B. W., Whitmore, B. C., Schweizer, F., Fall, S. M. 1997, AJ, 114, 2381

Moll, S. L., Mengel, S., de Grijs, R., Smith, L. J., \& Crowther, P. A. 2007, MNRAS, 382, 1877

Östlin, G., Cumming, R. J., \& Bergvall, N. 2007, A\&\&A, 461, 471

Oort, J. H. 1958, in: D. J. K. O'Connell (ed.), Proc. Ricerche Astronomiche, 5, 63

Ostriker, J. P., Spitzer Jr., L. J., \& Chevalier, R. A. 1972, ApJ (Letters), 176, L51

Parmentier, G., Goodwin, S. P., Kroupa, P., \& Baumgardt, H. 2008, ApJ, 678, 347

Pfalzner, S. 2009, A\&A (Letters), 498, L37

Portegies Zwart, S. F., McMillan, S. L. W., \& Gieles, M. 2010, ARA\&A, in prep.

Proszkow, E.-M., Adams, F. C., Hartmann, L. W., \& Tobin, J. J. 2009, ApJ, 697, 1020

Smith, L. J., Westmoquette, M. S., Gallagher III, J. S., O'Connell, R. W., Rosario, D. J., \& de Grijs, R. 2006, MNRAS, 370, 513

Spitzer Jr., L. J. 1940, MNRAS, 100, 396

Spitzer Jr., L. J. 1958, ApJ, 127, 17

Spitzer Jr., L. 1987, Dynamical evolution of globular clusters, Princeton: Princeton University Press

Spitzer Jr., L. J. \& Chevalier, R. A. 1973, ApJ, 183, 565

Theuns, T. 1991, Mem. Soc. Astron. Ital., 62, 909

Tutukov, A. V. 1978, A\&A, 70, 57

van den Bergh, S. 1957, ApJ, 125, 445

van den Bergh, S., \& McClure, R. D. 1980, A\& A, 88, 360

Vesperini, E. \& Heggie, D. C. 1997, MNRAS, 289, 898

Vesperini, E., Zepf, S. E., Kundu, A., \& Ashman, K. M. 2003, ApJ, 593, 760

Vesperini, E., McMillan, S. L. W., \& Portegies Zwart, S. 2009, ApJ, 698, 615

von Hoerner, S. 1958, Zeitschr. Astrophys., 44, 221

Whitmore, B. C., Chandar, R., \& Fall, S. M. 2007, AJ, 133, 1067

Whitmore, B. C., Zhang, Q., Leitherer, C., Fall, S. M., Schweizer, F., \& Miller, B. W. 1999, AJ, 118,1551

Wielen, R. 1971, A\&A, 13, 309

Wielen, R. 1988, in: J. E. Grindlay \& A. G. D. Philp (eds.), Proc. IAU Symp. No. 126, p. 393

Zepf, S. E., Ashman, K. M., English, J., Freeman, K. C., \& Sharples, R. M. 1999, AJ, 118, 752 